\title{
1 Principles of Structural Safety Studies
}

\subsection{Types of Extreme and Accidental Events}

Various structures and infrastructures are available for the purpose of sea-, air-, and landbased industrial applications, such as ships, offshore platforms, underwater vehicles, submarines, petrochemical plants, nuclear power plants, automobiles, airplanes, buildings, bridges, and box girder cranes.

Such structures and infrastructures are exposed to the actions and action effects of the operational and environmental conditions under which they are used. Although these effects are usually normal, they can be extreme or even accidental, with the latter leading to casualties, property damage, and environmental pollution.

The types of actions and action effects of the extreme conditions and accidents associated with structures and infrastructures can be classified as follows:

- Heating and cooling processes involved with flame cutting and welding in fabrication

- Extreme actions due to rogue waves and storms at sea

- Dynamic pressure due to the sloshing, slamming, and green water in ships

- Cold temperatures in the Arctic region

- Cold temperatures due to liquefied petroleum gas (LPG) cargos

- Cryogenic conditions due to liquefied natural gas (LNG) cargos

- Ultrahigh pressure due to subsea operations

- Ultrahigh pressure due to compressed gases such as compressed petroleum gas (CPG), compressed natural gas $(\mathrm{CNG})$ or compressed hydrogen gas $(\mathrm{CHG})$

- Elevated temperatures due to fire

- Springing and whipping due to abnormal waves or underwater explosions

- Blast pressure due to explosions or detonations

- Impact actions due to collisions

- Raking due to grounding by running over a hard object (e.g., rock) with a forward speed

- Penetration due to collisions or stranding

- Shaking, landslides, liquefaction, and tsunamis due to earthquakes

- Corrosion due to aging

- Cracking due to fatigue actions

- Denting due to the dropping of objects

The typical causes of extreme and accidental events on ships and offshore structures are shown in Figure 1.1. By nature, each action and action effect arises from a different source. However, all of them commonly cause structural safety issues (e.g., casualties, property damage, and environmental pollution). The mechanisms of the structural consequences of extreme conditions and accidents are highly nonlinear and non-Gaussian 
in association with multiple physical processes, multiple scales, and multiple criteria. Therefore, resolving the related issues is not straightforward.

It is essential for safety studies to implement advanced technologies and use more refined and sophisticated methodologies associated with probabilistic, risk-based, and limit state-based approaches. The application of multidisciplinary practices involving both advanced computational models and large-scale physical model testing is required.

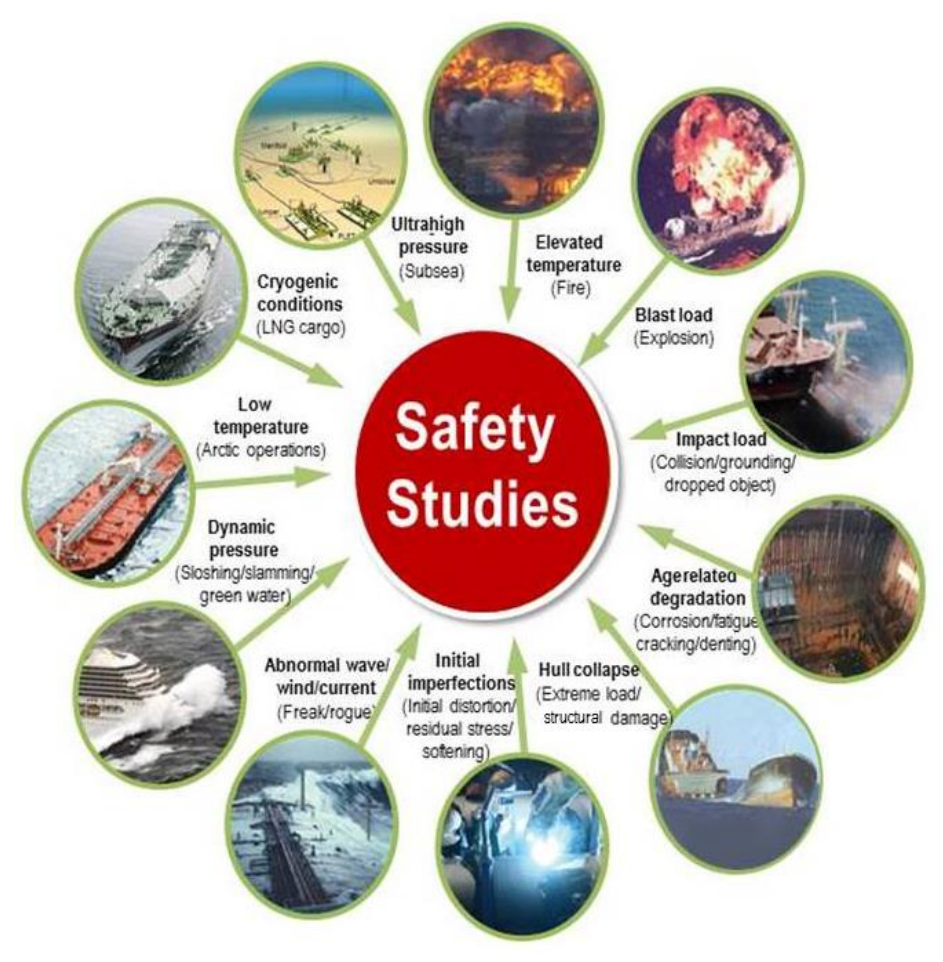

Figure 1.1. Extreme and accidental events involved in ships and offshore structures.

\subsection{Volatile, Uncertain, Complex, and Ambiguous Environments}

Extreme conditions and accidents are associated with volatility, uncertainty, complexity, and ambiguity. The structural consequences of extreme conditions and accidents are inevitably volatile, uncertain, complex, and ambiguous (VUCA):

1. They are volatile because the nature and dynamics of change are various, significant, frequent, and rapid. A number of random variables are involved, they are temporally and spatially changing, and their characteristics are dynamic or sometimes impulsive and happen over a very short period of time.

2. They are uncertain because hazards and their consequences are hard to forecast. Random variables cannot be defined with $100 \%$ certainty.

3. They are complex to analyze because they are affected by a number of factors that are highly nonlinear and do not necessarily follow Gaussian aspects involving multiple physical processes, multiple scales, or multiple criteria. 
4. They are ambiguous because they are hard to clarify and manifest due to the haziness of reality, the possibility of misreading them, and the difficulty of understanding situations.

To assess the integrity and safety of structural systems under extreme and accidental conditions in VUCA environments, advanced technologies should be applied via the smart processing of information from big databases that help clarify reality, which may be vague, unfamiliar, and dynamic. It is most important to prove that VUCA environments can be controlled and managed by advanced science and technology.

In structural safety studies conducted by the engineering community, mathematical modeling techniques are used to manage VUCA environments. Volatility can be managed through the relevance of random variables in mathematical models that represent a system's behavior. Uncertainty can be categorized as inherent uncertainty or modeling uncertainty. The inherent uncertainty caused by natural variabilities in environmental actions and material properties can be clarified by the statistical analysis of large databases. The modeling uncertainty arising from the inaccuracy of engineering modeling can be minimized using advanced computational models. Complexity can be managed via advanced computational models that suit the power of modern computer hardware and software. Ambiguity can be minimized by processing big databases, which can be obtained by collating historical events and/or by generating relevant artificial databases.

\subsection{Modeling of Random Parameters Affecting Structural Safety}

In structural safety studies, nonlinear structural responses under extreme and accidental conditions must be characterized. This can be done using the following nine-parameter function:

$$
f(X)=f\left(X_{1}, X_{2}, X_{3}, X_{4}, X_{5}, X_{6}, X_{7}, X_{8}, X_{9}\right)
$$

where $f(X)$ is the nonlinear function of structural responses, $X_{1}$ is the random parameter associated with geometric properties, $X_{2}$ is the random parameter associated with material properties, $X_{3}$ is the random parameter associated with fabrication-related initial imperfections, $X_{4}$ is the random parameter associated with loads in types or components, $X_{5}$ is the random parameter associated with loading speed, $X_{6}$ is the random parameter associated with temperature, $X_{7}$ is the random parameter associated with age-related degradation, $X_{8}$ is the random parameter associated with accidentinduced damage, and $X_{9}$ is the random parameter associated with human error.

Each of the individual random parameters presented in Equation (1.1) can further comprise multiple sub-parameters. The characteristics of the random parameters can be identified in a probabilistic manner using relevant probability density distribution functions.

\subsubsection{Geometric Properties}


Structures and infrastructures are composed of various types of structural members or elements. Plated structures, such as ships, ship-shaped offshore platforms, and box girder bridges or cranes, are composed of plates and support members. Framed structures are usually composed of beams, columns, and beam-columns. Typical examples of framed structures include fixed-type offshore structures and offshore jacket structures. Such framed structures are composed of tubular members in columns and braces, which are often dealt with in computational models as beams, columns, or beam-columns. Petrochemical plants and nuclear power plants are a combination of plated and framed structures. Airplane structures, underwater vehicles, and submarines are composed of thin shell or curved-plate elements with support members.

The dimensions (e.g., thickness) of a structural element are not perfectly identical over the member. For example, the thickness of a plate element may not be the same throughout the plate. This can be the result of mal-manufacturing or even prior design intention. However, the inaccuracy in today's modern manufacturing technologies of structural elements can be neglected with certainty. Furthermore, the dimensions of structural elements in engineering models may take the mean values as constants rather than as random variables.

\subsubsection{Material Properties}

The types of materials used for modern structures include metals (e.g., steel, aluminum alloys, and stainless steel) and composites. The typical parameters characterizing material properties include the elastic modulus (Young's modulus), Poisson's ratio, yield strength, ultimate tensile strength, and fracture strain. By nature, material properties are random variables in association with manufacturing quality. The mechanical properties of structural materials can be characterized by the stress-strain relation, which can be obtained by testing specimens. The nominal values of mechanical properties are provided by the materials' manufacturers. Regulatory bodies or classification societies specify the minimum requirements that material properties should meet for design robustness and the construction of structures.

Importantly, the structural responses analyzed using the nominal values of material properties must be different from those in reality. Furthermore, the probabilistic characteristics of mechanical properties in modern materials usually follow the Gaussian or normal function.

The details of material property modeling are provided by Paik et al. (2017) and Paik (2018a), for materials such as carbon steel, stainless steel, and aluminum alloy. For composite materials in polymer composites or composite laminates, the mechanical properties significantly depend on the types and manufacturing methods of the composites (Chung 2010, Grove 2018).

\subsubsection{Fabrication-Related Initial Imperfections}

Metal structures are typically fabricated by flame cutting and welding, processes that reach temperatures over $1,200{ }^{\circ} \mathrm{C}$. Therefore, they always have initial imperfections after fabrication in the form of distortions (deformations or deflections) and residual stresses, caused by the successive expansion and shrinkage that occurs during the heating and cooling processes. In a welded steel plate, tensile residual stress blocks are formed with a 
band width. The stress is approximately equal to the yield stress of the material because the molten metal can expand freely, as a liquid. After welding, it quickly reverts to its solid form. The shrinkage that occurs during cooling involves "plastic flow." Compressive residual stress is subsequently developed in the rest of the plating to achieve an equilibrium condition.

In the tensile residual stress blocks of welded aluminum structures, in contrast to welded steel structures, a softening phenomenon occurs in which the yield strength within the heat-affected zone is reduced relative to that of the base metal. As such fabrication-related initial imperfections may negatively affect the structural properties and load-carrying capacities of structures, they must be dealt with as parameters affecting structural design and strength assessment. The characteristics of fabrication-related initial imperfections are quite uncertain, but they usually follow the Gaussian or normal function.

The details of fabrication-related initial imperfection modeling in metallic materials are provided by Paik (2018a). For composite materials, reference can be made to Chung (2010) and Grove (2018).

\subsubsection{Types of Load}

The actions arising from environmental phenomena or operational conditions differ according to structural system type. For example, the actions on offshore structures are different from those on trading ships. The nature of offshore structures and their operations are such that waves, winds, and current (among other factors) are all involved. However, waves are the primary source of environmental action on trading ships at sea, where considerations related to specialized operations, such as berthing, are somewhat different (Hughes and Paik 2013).

The wave parameters for offshore structural designs include heights, periods, and directions with associated probabilities and persistence times. It is important to recognize that the waves that induce the most severe response in the global system structure may be different from those resulting in the maximum response in structural components. Furthermore, the response of floating offshore structures is wave-period dependent. Extreme waves often give rise to not only geometric nonlinearity (e.g., buckling), but also material nonlinearity (e.g., plasticity). More frequent waves rather than extreme waves govern fatigue life, although their magnitude may be smaller.

Wind is a primary metocean (meteollogical oceanographical) parameter that is important to the design of offshore structures that must withstand wind forces. This depends not only on the structural characteristics, such as windage area, but also on the speed and direction of the wind. For design, extreme wind speeds (i.e., with extreme gust values) for specified return periods must be obtained and specified with averaging times ranging from 3 seconds to 24 hours. Wind speeds are usually estimated at a standard height of $10 \mathrm{~m}$ above mean sea level, with corrections to more specific values at other heights. In addition, the spectra of fluctuating wind gusts are necessary, as wind gusts can excite resonant oscillations of offshore structures. Wind can cause various phenomena, such as vortex shedding and the associated vibrations in the flare towers of offshore platforms. 
Currents can affect the orientation of floating offshore structures. Therefore, they directly and indirectly affect both the short-term and long-term loads imposed on these structures and their mooring systems. Currents can increase the hull drag forces beyond the force of the wave system alone. They also ultimately affect the station-keeping of floating offshore structures and the performance of thrusters where used. Currents can also be the sources of vortex-induced vibrations in subsea risers and pipelines.

The types of load that structures and infrastructures are exposed to can be classified into dead loads, live loads, environmental loads, and accidental loads. Dead loads and live loads are associated with functional or operational services. They are usually modeled as static actions, where the effect of loading speed is neglected. Environmental loads arise from wind, waves, currents, snow, and earthquakes. They are time-variant and repeated in a cyclic pattern. In structural safety studies, the maximum values of environmental loads are often a basis of the safety criteria as static actions, whereas repeated aspects are of interest in the safe performance associated with fatigue fracture. If the magnitude of cyclic loads is large, low cycle fatigue can occur at a total number of load cycles less than $10^{4}$ over a period of a few hours, months, or several years. High cycle fatigue is associated with a smaller cyclic load at a total number of load cycles greater than $10^{4}$.

The probabilistic characteristics of environmental loads usually follow a non-Gaussian function. Furthermore, structural elements are probably subjected to the multiple components of actions and action effects. For example, the plate panels in plated structures, such as ships and ship-shaped offshore platforms, are subjected to longitudinal normal stress, transverse normal stress, shear stress, longitudinal in-plane bending stress, transverse in-plane bending stress, and lateral pressure. Although the maxima of the various types of load components are not always applied simultaneously, multiple load components usually coexist and interact.

Accidental loads arise from accidents, such as collisions, grounding, dropped objects, fires, and explosions. They are dynamic, having impact where the effect of strain rates cannot be neglected. The probabilistic characteristics of accidental loads usually follow a non-Gaussian function.

\subsubsection{Loading Speed}

Loading conditions depend on types of structures, among other factors. For example, loading conditions of offshore installations are different from those of trading ships (Paik and Thayamballi 2007).

In ships and offshore structures, sloshing, slamming, and green water are the typical sources of dynamic pressure loads that cause dynamic or impact issues associated with strain rate effects. Sloshing happens on partially filled tanks and is produced by accelerations arising from the motions of ships and ship-shaped offshore platforms at sea. The resonance between the natural sloshing period of the tank with liquids and the roll or pitch periods of the structure is of concern. Slamming and wave-slap impacts may also cause structural damage such as buckling, involving plasticity and dynamic effects. Green water refers to unbroken waves overtopping the bow, side, or stern structures of ships and offshore structures. The occurrence of green water depends on various factors, such as the relative motion between the structures and waves, the speed, the freeboard, 
and the harshness of the environment, exceeding the available freeboard. The green water problem can be an important design issue under harsh environmental conditions, as it can cause damage to deck houses, deck-mounted equipment (e.g., switch room compartments), watertight doors, walk-way ladders, and cable trays.

Structural systems, such as ships and offshore structures, can be subjected to impact loads arising from collisions, grounding, or dropped objects. Collision is a phenomenon in which side, bow, or stern structures are damaged by colliding with an object. Collision accidents are associated with impact issues because they involve two colliding objects, where one or both are moving at speed. For example, collisions can occur between two automobiles, between two ships, between a ship and an offshore platform or a bridge pier, between a ship and an iceberg, or between an offshore platform and an iceberg.

Grounding is a phenomenon in which bottom structures are damaged by some accidental sources. Three types of grounding accidents are relevant, namely grounding with a forward speed, stranding, and squatting. Grounding with a forward speed occurs when an obstruction deforms the bottom inward and/or enters into and cuts, for example, through the trading ship structure as the vessel moves forward on a rock. This type of damage is called a raking damage and forms a very long gash on the bottom of structures. Stranding occurs when the powered structure is out of control, demonstrates engine failure, and incurs bottom damage or penetration mostly in the depth direction (e.g., as a result of hydrodynamic instability with tidal differences in ships). A stranding situation is similar to a collision, such that the side that is struck is subjected to mainly out-of-plane impact. Squatting occurs when an object moves in shallow water, where bottom plates may deform due to the difference between the external and internal pressures, as the external pressure in rapid water particles can decrease.

The deck plates of structures and infrastructures may be subjected to impacts due to objects dropped from cranes resulting in damage. Such mechanical damage can lead to denting, cracking, and residual stresses or strains, with plastic deformation or coating damage causing pit corrosion.

Explosions due to hydrocarbons, such as oil and gas, occur due to hydrocarbon leakage and ignition. Explosions cause a blast or a rapid increase in overpressure loads and drag forces. Within the framework of risk assessment and management, the action effects of overpressure and drag forces are the primary hazard-related concerns surrounding explosions. Both plasticity and strain-rate sensitivity issues are always involved with nonlinear structural responses to blast pressure loads.

Three differences between static/quasi-static and dynamic/impact loading cases are usually relevant (Paik 2018a). The first difference concerns the stress field. In an impact loading situation, tensile stresses can occur even under compressive far-field loading. Furthermore, stress concentrations can occur even without notches. The second difference is that the dynamic/impact structural behavior can vary as a function of the strain rate. The first and second differences always interact. The third difference regards the failure pattern. Under impact loading, brittle fracture is a greater possibility for steels and aluminum alloys that are predominantly ductile under static/quasi-static loading. Specifically, the energy absorption capacity by ductile yielding decreases at high strain rates after an increase in yield strength, but after a decrease in fracture strain. 
The profile of dynamic or impact actions is generally characterized by four parameters: (1) rise time until the peak load is reached, (2) peak load, (3) load decay type beyond the peak load, and (4) load duration. For practical engineering purposes, the problem of dynamic or impact actions in terms of structural response may be idealized within three domains depending on the ratio of the duration (t) of dynamic or impact actions to the natural period $(\mathrm{T})$ of the structure:

- Quasi-static domain, when $c_{1} \leq t / T$

- Dynamic or impact domain, when $c_{2} \leq t / T<c_{1}$

- Impulsive domain, when $t / T<c_{1}$

The above criteria may be interpreted as $c_{1}=3$ and $c_{2}=0.3$ (NORSOK 1999). Moderate or high strain rates are involved in some types of loads depending on loading speed and should be considered in structural safety studies (Jones 2012, Paik 2018a). For example, the duration of blast pressure loads in explosions is a few milliseconds.

The structural responses in high speed loading or impact loading are entirely different from those in static or low speed loading. Material properties are significantly affected by loading speed or strain rates. As strain rate increases, the material yield stress of metals tends to increase but their fracture strain tends to decrease. Another issue to be resolved in structural safety studies is that the strain-rate characteristics of different structural elements may differ, as the loading speed over individual elements may differ. Furthermore, when numerical computations using nonlinear finite element methods are applied in structural crashworthiness analyses, dynamic fracture strain must be properly defined before the computations.

Many uncertainties and ambiguities are pertinent, as strain-rate effects cannot be perfectly manifested in association with material properties, such as yield stress and critical fracture strain. The details of strain-rate effects on mechanical properties are provided by Jones (2012), Paik et al. (2017), and Paik (2018a) for some materials, such as carbon steel, stainless steel, and aluminum alloy.

\subsubsection{Temperature}

Structures and infrastructures are subjected to lower and higher temperatures than room temperature due to environmental and operational conditions of service. Furthermore, the temperatures on land differ from those at sea. The probable extremes of sea surface temperature are sometimes more severe than the corresponding 50-year return period temperatures. The extremes of sea surface temperature also occur less frequently than air temperature extremes. Sea temperature information is important for fracture toughness engineering in Arctic operations, where the average winter temperature is $-40{ }^{\circ} \mathrm{C}$ and the lowest is reportedly $-68{ }^{\circ} \mathrm{C}$. Year-round temperatures and humidity are also of interest in association with corrosion damage.

The liquefaction of natural gas can be achieved by either lowering temperature or increasing atmospheric pressure, with the former technique being adopted more often in current industrial applications. Temperature should be lowered to $-163{ }^{\circ} \mathrm{C}$ to process LNG and to $-43{ }^{\circ} \mathrm{C}$ to process LPG. The mechanical properties of materials are 
significantly affected by low temperature. Furthermore, those used for the cargo tank structures of LNG carriers or of LNG floating, production, storage, and offloading units can be exposed to cryogenic conditions, caused by an accidental leakage of LNG. As such, it is very important to manage brittle fracture issues in designing and engineering structures for cryogenic conditions.

In offshore oil and gas installations, more than $70 \%$ of accidents stem from hydrocarbon explosions and fires. Hydrocarbons can explode through ignition when combined with an oxidizer (usually oxygen or air). Combustion occurs when temperatures increase to the point at which hydrocarbon molecules react spontaneously with the oxidizer. Fire is a combustible vapor or gas that combines with an oxidizer in a combustion process that manifests in the evolution of light, heat, and flames.

Within the framework of risk assessment and management, the action effects of elevated temperatures are the primary hazard-related concerns surrounding fire. The thermal characteristics of steel are the main factors affecting structural integrity in fires (Franssen and Real 2010). The specific heat of steel varies with temperature. At temperatures above $400{ }^{\circ} \mathrm{C}$, the mechanical properties of steel decrease significantly. The heat from fire flows relatively rapidly in steel, which is a good heat conductor compared to other materials (e.g., concrete). Thus, fire can lead to the collapse of steel structures (involving plasticity) and the severity of fire loads usually requires the application of safety barriers, such as passive fire protection, for critical structural elements.

The mechanical properties of materials are significantly affected by temperatures. The details of the effects of cold temperatures on mechanical properties are provided by Paik et al. (2017) and Paik (2018a) for some materials, such as carbon steel, stainless steel, and aluminum alloy.

\subsubsection{Age-Related Degradation}

The typical types of deterioration involved in aged structures and infrastructures include corrosion, fatigue cracks, coating breakdown, and out-of-tolerance misalignments (Paik and Melchers 2008). Corrosion can either be general (uniform) corrosion or localized corrosion. Cracking may be caused by fatigue due to dynamic actions arising from environmental phenomena, operations, and other causes, such as high local stresses and hot spots. Cold temperature exposure may make the material brittle. Given the appropriate high stress-strain rates, this can result in brittle fractures. However, with attention now being paid to selecting materials with good toughness, brittle fracture is much less common. Inadequate fabrication can result in significant initial defects and misalignments, which may also increase the probability of fatigue cracking.

The mechanism of age-related degradation is uncertain, complex, and essentially timevariant. Corrosion wastage and fatigue cracking are often interrelated, as cracking damage can initiate and evolve from corrosion pits. The progress of corrosion depends on the corrosion environment and operational conditions.

\subsubsection{Accident-Induced Damage}

Accidents, such as fires, explosions, collisions, grounding, and dropped objects, occur continuously in structures and infrastructures, despite continued efforts to prevent them. Accident-induced damage may involve nonlinear action effects at the material, structural 
element, or entire structural system level in association with geometric and mechanical properties. Advanced computational models are needed to characterize accident-induced damage, which follows a non-Gaussian function. Safety studies should be conducted quickly and accurately to facilitate the rapid planning of salvage and rescue operations for structural systems immediately following accidents and to expedite the determination of the location and extent of damage, even if only approximately. The residual strength characteristics of damaged structures are highly nonlinear and probabilistic.

\subsubsection{Human Error}

Human error is the key cause underlying most of the accidents in industry. It is defined as a situation in which something has been done without intention, expectation, or desirability (Petersen 1996). Human error can happen when human actions fail to achieve their goal because the original plan is inadequate, leading to mistakes despite working as planned, or because of inadequate performance, leading to deviations despite the original plan being adequate.

Most accidents are the result of a long chain of human error (Paik 2012). Unless human error can be minimized, it is not possible to fully protect human health and the environment or to ensure safety. Human error results from ignoring human factors and ergonomics, with such ignorance either being the root cause of or a major contributing factor to many accidents. Ignorance of the engineering factors that cause human error is primarily due to a lack of knowledge and guidance in the design, building, and operation stages of structural systems.

To eliminate or reduce human error, human factors and ergonomics must be fully accounted for, with more extensive knowledge applied to all stages, from design and engineering to building and operation. A key part of this more extensive knowledge consists of lessons learned from past accidents, with a focus on structural safety studies.

However, in the current engineering models used in safety studies, it is quite challenging to account for the effects of human error. Classifying judgments as either adequate or inadequate may be a harmful oversimplification of the mechanisms associated with extreme and accidental events, which are volatile, uncertain, complex, and ambiguous. The most desirable approach to managing human error is to take advantage of digital technologies for decision making rather than using human experience or insight.

\subsection{Limit States and Risks}

One of the traditional approaches for structural safety assurance is to keep the working stresses from the design loads under a certain level which is called the allowable stress. In this case, the structural safety criterion is typically expressed as follows:

$$
\sigma<\sigma_{a}
$$

where $\sigma$ is the working stress and $\sigma_{a}$ is the allowable stress which is usually specified as some fraction of mechanical properties of material such as yield strength. It is obvious that Equation (1.2) does not represent nonlinear aspects of structures and infrastructures associated with extreme conditions and accidents. 
Limit states and risks are the best basis of structural safety studies associated with extreme and accidental events (Lee and McCormick 2011, Rougier et al. 2013, Paik 2018a). A limit state indicates a condition under which a particular structural element or an entire structural system would fail to perform its intended function. Four types of limit states are relevant to structures (Paik 2018a):

- The ultimate limit state (ULS)

- The accidental limit state (ALS)

- The fatigue limit state (FLS)

- The serviceability limit state (SLS)

The ULS is the structural collapse that results from the loss of structural capacity in terms of stiffness and strength that typically arises from the buckling and plastic collapse of structural components under extreme loads. The ALS is the excessive structural damage that results from accidents, such as collisions, grounding, explosions, and fireall of which affect the safety of the structure, the environment, and the personnel. The FLS is the fatigue cracking of structural details that results from stress concentration and damage accumulation under repeated loading actions. The SLS represents the failure in normal operations that results from deterioration in routine functionality. Typical examples of the SLS include local damage, unacceptable deformation, and excessive vibration and noise affecting the proper functioning of structural elements or equipment.

The partial safety factor-based criterion is usually adopted for the structural safety studies associated with limit states (as described in Chapter 3):

$$
C_{d}>D_{d}
$$

where $C_{d}=C_{k} / \gamma_{C}$ is the design capacity (strength), $D_{d}=\gamma_{D} D_{k}$ is the design demand (loads), $C_{k}$ is the characteristic value of capacity, $D_{k}$ is the characteristic value of demand, $\gamma_{C}$ is the partial safety factor associated with capacity, and $\gamma_{D}$ is the partial safety factor associated with demand.

A number of hazards must be dealt with in the process of risk assessment. Risk exists wherever potential hazards exist. In the engineering community, the term "risk" is typically defined as either the product or a composite of two factors. The first factor is the probability or likelihood of any accident or limit state leading to severe consequences, such as human injuries, environmental damage, and loss of property or financial exposure, occurring. The second factor is the resulting consequences, and thus the risk-based criterion is expressed as follows:

$$
R=F^{a} \times C^{b}<R_{a}
$$

where $R$ is the risk, $F$ is the frequency of the hazard, $C$ is the level of consequence, and $a$ and $b$ are ascending constants, which are often interpreted as $a=b=1$. To weight the consequence, $a=1$ and $b=2$ may also be used. $R_{a}$ is the acceptable level of risk (acceptance criterion).

The frequency of the hazard indicates how often the hazard takes place. It is usually measured by the number of occurrences per unit time (e.g., per year), considering that the design life of structures and infrastructures is usually several decades (e.g., 25 years for 
ships, 40 years for offshore platforms, 40 years for nuclear power plants, and 100 years for box girder bridges). The level of consequence indicates the severity and impact of the consequence. It is sometimes measured monetarily (e.g., repair costs for accidental damage, recovery costs for environmental pollution, or insurance costs for pollution). For human injuries or casualties, the number of people involved in an event can be a measure of the consequence. The risk is then assessed in association with casualties, property damage, and environmental pollution.

In a qualitative risk assessment approach, $F$ and $C$ are often determined by simple methods that are based on past experiences or insights, without application of refined computations or physical model testing. For example, $F$ may be scored between 1 (rare occurrence) and 5 (very frequent occurrence) and $C$ may be scored between 1 (very minor damage) and 5 (catastrophic damage). Substituting $a=b=1$ in Equation (1.4), the minimum and maximum risk values become 1 and 25, respectively. For example, the acceptable level of risk can be defined as 5. In this case, it may be said that the risk is tolerable if the calculated risk is smaller than 5. Otherwise, the risk level is not acceptable for safety and must be reduced. Although this simple approach may be better than the existing prescriptive approaches, it is not good enough to resolve the issues associated with extreme conditions and accidents in VUCA environments.

The acceptance criteria of risk may vary from one industry to another depending on various factors such as the type of accident, the seriousness of its consequence or public and media sensitivity. Appropriate criteria of risk acceptance are not readily available, while the ALARP (as-low-as-reasonably-practicable) principle may be applied.

In practice, the acceptable level of risk can be selected on the basis of successful prior experience where the risk levels of existing successful structures and infrastructures against extreme conditions and accidents are assessed by making use of advanced safety studies with limit states and risks that apply quantitative approaches incorporating advanced computational models and large-scale or full-scale physical model testing. Economic value analysis is also implemented to minimize total expected costs during the service life of structures and infrastructures. The acceptable level of risk so selected can then be employed for the new types of structure and infrastructure.

\subsection{Future Trends Toward Advanced Structural Safety Studies}

Limit state- and risk-based approaches are the best methods for assessing and managing the challenging issues associated with hazards and their consequences, considering that VUCA environments are inevitably involved in extreme and accidental events. Successful design and engineering should not only meet functional requirements, but also health, safety, environment, and ergonomics (HSE\&E) requirements. Functional requirements represent operability under normal conditions. The HSE\&E requirements address safe performance and integrity in extreme and accidental conditions. The paradigm shift in design and engineering is now pertinent for structures and infrastructures, as described in Figure 1.2. Paik (2015) suggested that the International Maritime Organization's (IMO's) original concept be modified to achieve goal-based standards (GBS) by adding a new tier explicitly dealing with the HSE\&E requirements, as shown in Figure 1.3. 
To minimize risk, one may attempt to reduce the likelihood of undesirable events or hazards occurring; to contain, reduce, or mitigate the consequences of undesirable events or hazards; or both. In the lifecycle of structures and infrastructures, limit states and risks must be assessed, managed, and controlled so that they remain at tolerable levels. Risk management and control should be an on-going process throughout the lifecycle of structural systems. The different lifecycle stages offer different opportunities for risk control and management.

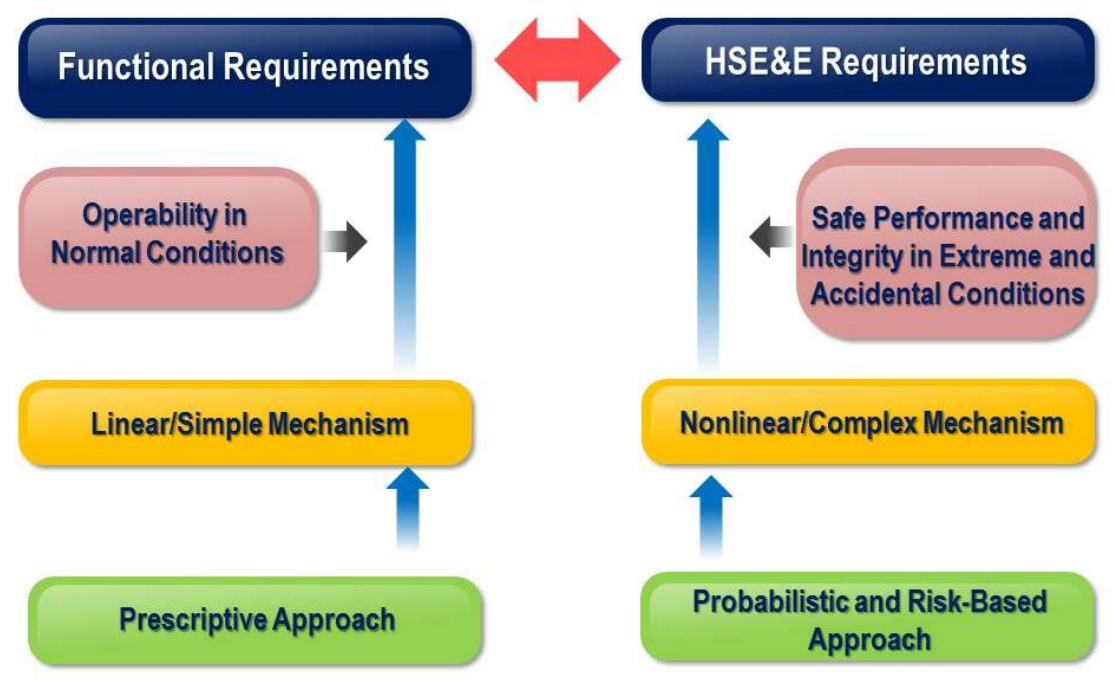

Figure 1.2. Paradigm shift for successful design and engineering.

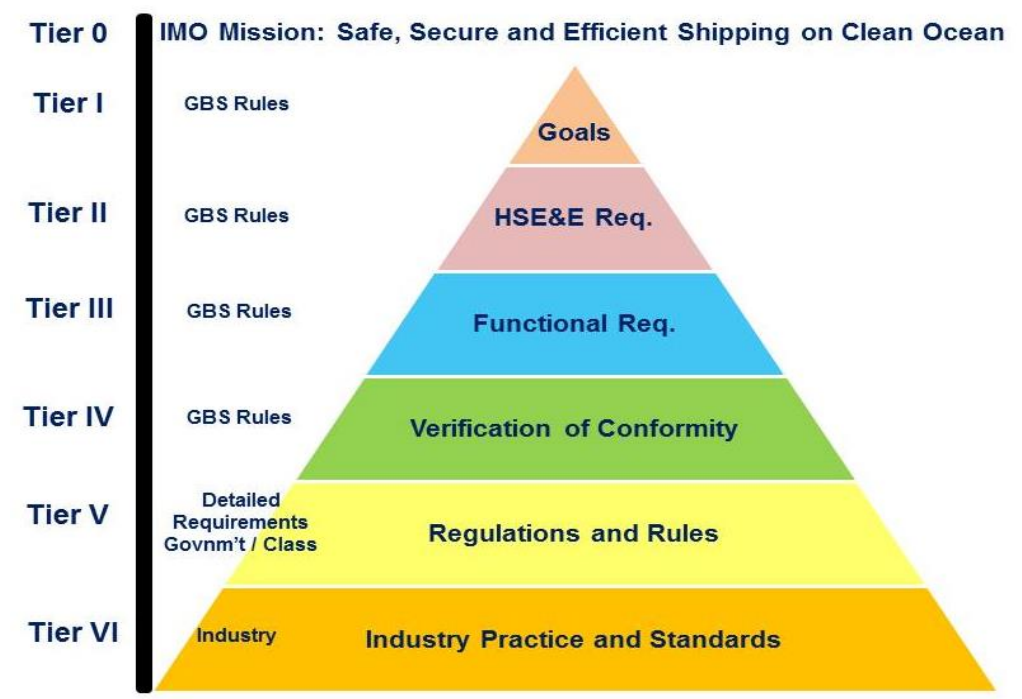

Figure 1.3. Modifications to the IMO's original concept of GBS with six tiers suggested by Paik (2015). 
In this regard, integrated and multidisciplinary approaches should be applied, as shown in Figure 1.4 (Paik 2015). Computation alone is insufficient for identifying all aspects. Physical model testing using full-scale prototypes or at least large-scale models is essential, as scaling and similarity laws for the conversion of small-scale (toy) model test results into real, full-scale structural systems are not usually available for extreme conditions and accidents. In this case, relevant physical model testing facilities are required to meet the needs of using full-scale or at least large-scale physical models as described in Chapter 18 (Paik 2014, 2018b). Operations must be monitored to provide feedback to a service database relevant to the design stage. Lastly, engineers should be well trained in and familiar with computation, physical model testing, and operations.

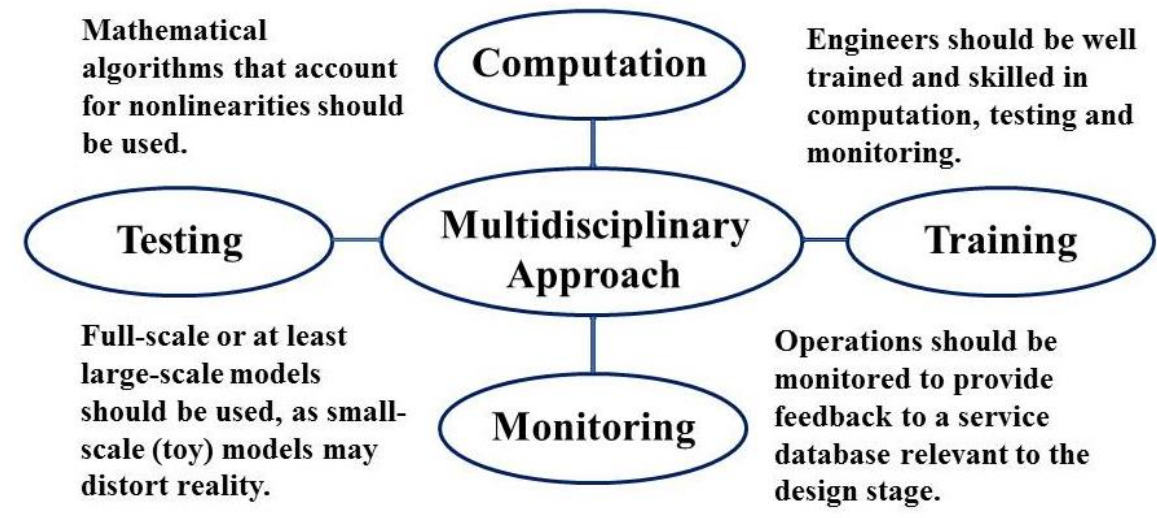

Figure 1.4. Multidisciplinary approach to meeting the HSE\&E requirements.

\section{References}

Chung DDL (2010). Composite materials - science and applications. $2^{\text {nd }}$ Edition, Springer, London, U.K.

Grove S (2018). Composite materials and structures (for engineering students). Kindle Direct Publishing, Amazon Publishing, Seattle, WA, U.S.A.

Hughes OF, Paik JK (2013). Ship structural analysis and design. The Society of Naval Architects and Marine Engineers, Alexandria, VA, U.S.A.

Jones N (2012). Structural impact. $2^{\text {nd }}$ Edition, Cambridge University Press, New York, NY, U.S.A.

Lee JC, McCormick NJ (2011). Risk and safety analysis of nuclear systems. John Wiley \& Sons, Chichester, U.K.

NORSOK (1999). Design of steel structures. Norwegian Standards, Norway.

Paik JK (2012). Lessons learned - what maritime accidents can teach us about human error, and structural design and engineering. Marine Technology, The Society of Naval Architects and Marine Engineers, Alexandria, VA, U.S.A., April, 62-66.

Paik JK (2014). Putting deep water safety to the test - anticipating and testing for extreme and accidental events. Marine Technology, The Society of Naval Architects and Marine Engineers, Alexandria, VA, U.S.A.

Paik JK (2015). Making the case for adding variety to goal-based standards. The Naval Architect, The Royal Institution of Naval Architects, London, U.K., January, 22-24. 
Paik JK (2018a). Ultimate limit state analysis and design of plated structures. $2^{\text {nd }}$ Edition, John Wiley \& Sons, Chichester, U.K.

Paik JK (2018b). A global R\&D hub for advanced safety studies. The Naval Architect, The Royal Institution of Naval Architects, London, U.K., 35-37.

Paik JK, Kim KJ, Lee JH, Jung BG, Kim SJ (2017). Test database of the mechanical properties of mild, high-tensile and stainless steel and aluminum alloy associated with cold temperatures and strain rates. Ships and Offshore Structures, 12(S1): S230-S256.

Paik JK, Melchers RE (2008). Condition assessment of aged structures. CRC Press, New York, NY, U.S.A.

Paik JK, Thayamballi AK (2007). Ship-shaped offshore installations: design, building, and operation. Cambridge University Press, New York, NY, U.S.A.

Petersen D (1996). Human error reduction and safety management. $3^{\text {rd }}$ Edition, Van Nostrand Reinhold, New York, NY, U.S.A.

Rougier J, Sparks S, Hill L (2013). Risk and uncertainty assessment for natural hazards. Cambridge University Press, New York, NY, U.S.A. 